1. Mellor, A.L., and Munn, D.H. 2000. Immunology at the maternal-fetal interface: lessons for $\mathrm{T}$ cell tolerance and suppression. Annu. Rev. Immunol. 18:367-391.

2. Hunt, J.S., and Pollard, J.W. 1992. Macrophages in the uterus and placenta. Curr. Top. Microbiol. Immu nol. 181:39-63.

3. Dimitriadis, E., White, C.A., Jones, R.L., and Salamonsen, L.A. 2005. Cytokines, chemokines and growth factors in endometrium related to implantation. Hum. Reprod. Update. 11:613-630.

4. Pollard, J.W., et al. 1987. Apparent role of the macrophage growth factor, CSF-1, in placental development. Nature. 330:484-486.

5. Robertson, S.A., Mayrhofer, G., and Seamark, R.F. 1992. Uterine epithelial cells synthesize granulocyte-macrophage colony-stimulating factor and interleukin-6 in pregnant and nonpregnant mice. Biol. Reprod. 46:1069-1079.

6. Chitu, V., and Stanley, E.R. 2006. Colony-stimulating factor-1 in immunity and inflammation. Curr. Opin. Immunol. 18:39-48.

7. Plaks, V., et al. 2008. Uterine DCs are crucial for decidua formation during embryo implantation in mice. J. Clin. Invest. 118:3954-3965.

8. Pollard, J.W., Hunt, J.S., Wiktor-Jedrzejczak, W., and Stanley, E.R. 1991. A pregnancy defect in the osteopetrotic $(O P / O P)$ mouse demonstrates the requirement for CSF-1 in female fertility. Dev. Biol. 148:273-283.

9. Blois, S.M., et al. 2007. Dendritic cells: key to fetal tolerance? Biol. Reprod. 77:590-598.

10. Rockwell, L.C., Pillai, S., Olson, C.E., and Koos, R.D. 2002. Inhibition of vascular endothelial growth factor/vascular permeability factor action blocks estrogen-induced uterine edema and implantation in rodents. Biol. Reprod. 67:1804-1810.

11. Travis, M.A., et al. 2007. Loss of integrin alpha(v)beta 8 on dendritic cells causes autoimmunity and colitis in mice. Nature. 449:361-365.

12. Aluvihare, V.R., Kallikourdis, M., and Betz, A.G. 2004. Regulatory T cells mediate maternal tolerance to the fetus. Nat. Immunol. 5:266-271.

13. Ashkar, A.A., and Croy, B.A. 1999. Interferongamma contributes to the normalcy of murine pregnancy. Biol. Reprod. 61:493-502.

14. Condon, J.C., Jeyasuria, P., Faust, J.M., and Mendelson, C.R. 2004. Surfactant protein secreted by the maturing mouse fetal lung acts as a hormone that signals the initiation of parturition. Proc. Natl. Acad. Sci. U. S. A. 101:4978-4983.

15. Qiu, X., Zhu, L., and Pollard, J.W. Colony stimu- lating factor-1-dependent macrophage functions regulate the maternal decidua immune responses against Listeria monocytogenes infections during early gestation in mice. Infect. Immun. doi:10.1128/ IAI.01022-08

16. Ain, R., Trinh, M.L., and Soares, M.J. 2004. Interleukin-11 signaling is required for the differentiation of natural killer cells at the maternal-fetal interface. Dev. Dyn. 231:700-708.

17. Bilinski, P., Roopenian, D., and Gossler, A. 1998. Maternal IL-11Ralpha function is required for normal decidua and fetoplacental development in mice. Genes Dev. 12:2234-2243.

18. Hanna, J., et al. 2006. Decidual NK cells regulate key developmental processes at the human fetalmaternal interface. Nat. Med. 12:1065-1074.

19. Barber, E.M., and Pollard, J.W. 2003. The uterine NK cell population requires IL-15 but these cells are not required for pregnancy nor the resolution of a Listeria monocytogenes infection. J. Immunol. 171:37-46.

20. Pollard, J.W. 2004. Tumor educated macrophages promote tumor progression and metastasis. Nat. Rev. Cancer. 4:71-78.

21. Levine, R.J., et al. 2006. Soluble endoglin and other circulating antiangiogenic factors in preeclampsia. N. Engl. J. Med. 355:992-1005.

\title{
Tumor metabolism: cancer cells give and take lactate
}

\author{
Gregg L. Semenza
}

Vascular Program, Institute for Cell Engineering, Departments of Pediatrics, Medicine, Oncology, and Radiation Oncology, and McKusick-Nathans Institute of Genetic Medicine, Johns Hopkins University School of Medicine, Baltimore, Maryland, USA.

Tumors contain well-oxygenated (aerobic) and poorly oxygenated (hypoxic) regions, which were thought to utilize glucose for oxidative and glycolytic metabolism, respectively. In this issue of the JCI, Sonveaux et al. show that human cancer cells cultured under hypoxic conditions convert glucose to lactate and extrude it, whereas aerobic cancer cells take up lactate via monocarboxylate transporter 1 (MCT1) and utilize it for oxidative phosphorylation (see the related article, doi:10.1172/JCI36843). When MCT1 is inhibited, aerobic cancer cells take up glucose rather than lactate, and hypoxic cancer cells die due to glucose deprivation. Treatment of tumor-bearing mice with an inhibitor of MCT1 retarded tumor growth. MCT1 expression was detected exclusively in nonhypoxic regions of human cancer biopsy samples, and in combination, these data suggest that MCT1 inhibition holds potential as a novel cancer therapy.

The pioneering work of Peter Vaupel and his colleagues established that the partial pressure of oxygen $\left(\mathrm{pO}_{2}\right)$ within human cancers is frequently much lower than that of the surrounding normal tissue and that intratumoral hypoxia is associated with an increased risk of local spread, metastasis, and patient mortality (1). Rakesh Jain's

Nonstandard abbreviations used: LDH, lactate dehydrogenase; MCT, monocarboxylate transporter.

Conflict of interest: The author has declared that no conflict of interest exists.

Citation for this article: $J$. Clin. Invest. doi:10.1172/ JCI37373. laboratory demonstrated that in mouse tumor xenografts, the mean $\mathrm{pO}_{2}$ and $\mathrm{pH}$ declined as distance from the nearest blood vessel increased (2), reflecting the switch from oxidative to glycolytic metabolism that occurs in response to reduced $\mathrm{O}_{2}$ availability.

This metabolic reprogramming is orchestrated by HIF-1 through the transcriptional activation of key genes encoding metabolic enzymes, including: $L D H A$, encoding lactate dehydrogenase $\mathrm{A}$, which converts pyruvate to lactate (3); $P D K 1$, encoding pyruvate dehydrogenase kinase 1 , which inactivates the enzyme responsible for conversion of pyruvate to acetyl-CoA, thereby shunting pyruvate away from the mitochondria $(4,5)$; and BNIP3, which encodes a member of the BCL2 family that triggers selective mitochondrial autophagy (6) (Figure 1). In addition, HIF-1 transactivates GLUT1 (7) - which encodes a glucose transporter that increases glucose uptake to compensate for the fact that, compared with oxidative phosphorylation, glycolysis generates approximately 19-fold less ATP per mole of glucose - and genes encoding the glycolytic enzymes that convert glucose to pyruvate (3). The extracellular acidosis associated with hypoxic tumor cells is due to both increased $\mathrm{H}^{+}$production and increased $\mathrm{H}^{+}$ efflux through the HIF-1-mediated transactivation of: $C A 9$, which encodes carbonic anhydrase IX (8); MCT4, which encodes monocarboxylate transporter 4 (9); and NHE1, which encodes sodium-hydrogen exchanger 1 (10).

\section{Metabolic symbiosis}

In this issue of the JCI, the elegant article by Sonveaux, Dewhirst, et al. makes a major contribution to the field of cancer biology (11). 


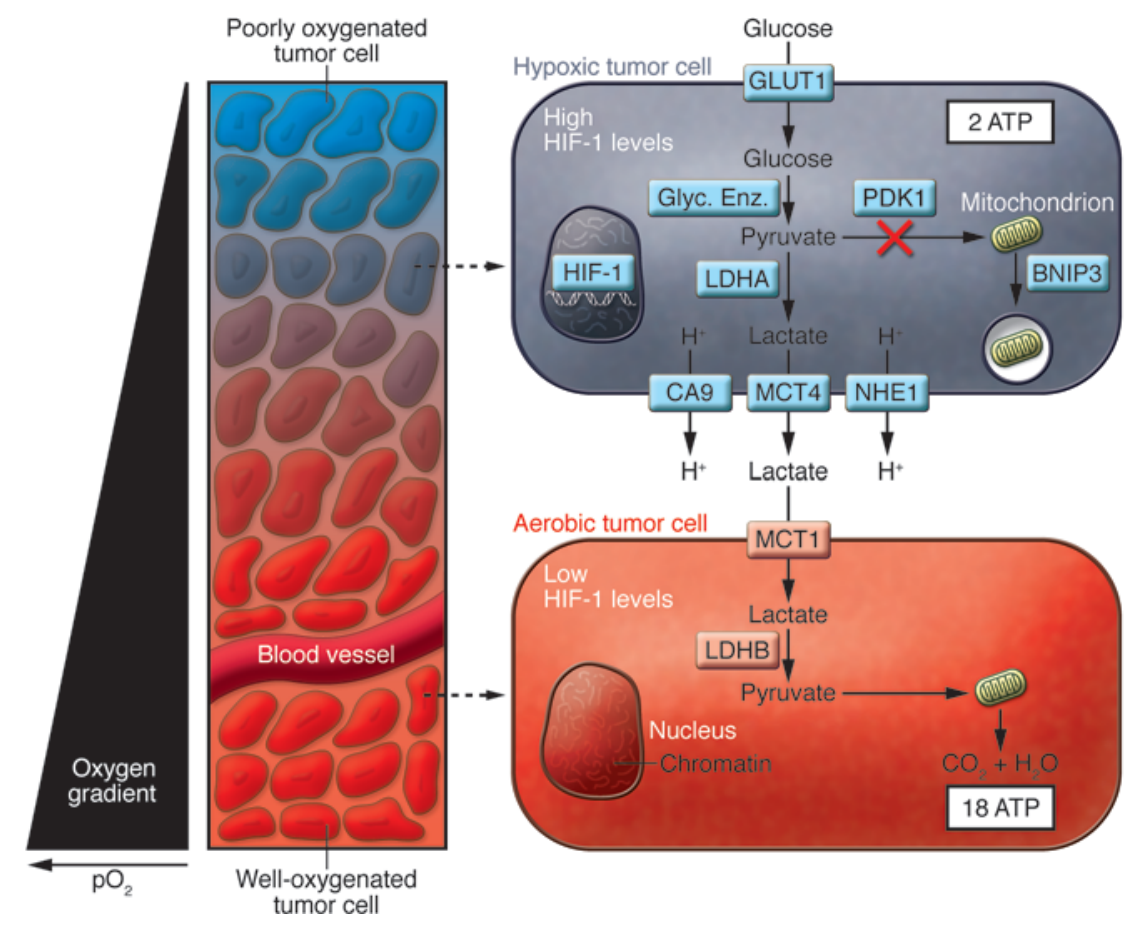

Figure 1

Intratumoral hypoxia and metabolic symbiosis. Tumors are characterized by gradients of $\mathrm{O}_{2}$ levels, based on the distance of tumor cells from a functional blood vessel. In the schematic, tumor cells surrounding the blood vessel are well oxygenated, whereas the tumor cells more distant from the vessel are poorly oxygenated and express high levels of HIF-1. HIF-1 induces the expression of proteins that increase: uptake of glucose (e.g., glucose transporter 1 [GLUT1]); conversion of glucose to pyruvate (e.g., glycolytic enzymes [Glyc. Enz.]); generation of lactate and $\mathrm{H}^{+}$(e.g., LDHA); and efflux of these molecules out of the cell (e.g., carbonic anhydrase IX [CA9], sodium-hydrogen exchanger 1 [NHE1], MCT4). Two moles of lactate are produced for each mole of glucose consumed by the hypoxic cell. This increase in glycolytic metabolism is associated with reduced substrate delivery to the mitochondria (through the action of pyruvate dehydrogenase kinase 1 [PDK1]) and reduced mitochondrial mass (as a result of autophagy triggered by BNIP3). As described by Sonveaux et al. in their study in this issue of the $\mathrm{JCl}(11)$, aerobic tumor cells express proteins that allow them to take up lactate (e.g., MCT1) and use it (e.g., LDHB), in the presence of $\mathrm{O}_{2}$, as their principal substrate for mitochondrial oxidative phosphorylation. Note that hypoxic cells generate $2 \mathrm{~mol}$ of ATP and $2 \mathrm{~mol}$ of lactate per mol of glucose consumed, whereas aerobic cells generate $36 \mathrm{~mol}$ of ATP per $2 \mathrm{~mol}$ of lactate consumed. $\mathrm{pO}_{2}$, partial pressure of oxygen.

The authors demonstrate the existence of a "metabolic symbiosis" between hypoxic and aerobic cancer cells, in which lactate produced by hypoxic cells is taken up by aerobic cells, which use it as their principal substrate for oxidative phosphorylation. As a result, the limited glucose available to the tumor is used most efficiently: hypoxic cells downregulate oxidative phosphorylation in order to maintain redox homeostasis $(4,6)$ and must consume large amounts of glucose to maintain energy homeostasis (12). At the molecular level, a key player in this symbiotic relationship is monocarboxylate transporter 1 (MCT1), which differs from MCT4 in two respects: the expression of MCT1 is hypoxia repressed rather than hypoxia induced, and it transports lactate into, rather than out of, cancer cells. $\mathrm{O}_{2}$ dependent expression of MCT1 allows aerobic cancer cells to efficiently take up lactate and, in concert with the $\mathrm{O}_{2}$-dependent expression of LDHB, to utilize lactate as an energy substrate, thereby freeing these cells from the need to take up large quantities of glucose (11). Thus, while Sonveaux et al. focused their attention on MCT1, it is important to note that $\mathrm{O}_{2}$ regulates the expression of many key enzymes in these metabolic pathways (Figure 1). It will be interesting to determine whether HIF-1 also controls MCT1 and LDHB expression, perhaps by inducing expression of a transcriptional repressor, as has been described for the $\mathrm{O}_{2}$-dependent regulation of adipogenesis (13).
Was there any precedent that should have alerted us to the existence of this symbiotic relationship between aerobic and hypoxic cancer cells (11)? Of course: the well-known recycling of lactate in exercising muscle. Just as tumors co-opt physiological mechanisms regulating vascularization, which are orchestrated by HIF-1 through transactivation of genes encoding VEGF, stromalderived factor 1 , and other angiogenic factors (14), so do they modulate metabolism through a program that functions to efficiently distribute glucose and lactate to fast-twitch (glycolytic) and slow-twitch (oxidative) muscle fibers.

The ability of tumor cells to adapt to regional variation (i.e., spatial heterogeneity) of oxygenation is remarkable. In another recent publication, Cárdenas-Navia, Dewhirst, and colleagues have also contributed to a large body of literature indicating that a cancer cell that is hypoxic at one moment may be aerobic an hour later and vice versa (15). In other words, there appears to be cyclic variation (i.e., temporal heterogeneity) in oxygenation. This, in turn, implies dynamic regulation of the metabolic symbiosis, such that cells may cycle between lactateproducing and lactate-consuming states.

\section{Therapeutic implications}

Hypoxic tumor cells are preferentially resistant to chemotherapy and radiation, thereby leading to treatment failure or disease relapse and, ultimately, to patient mortality (1). In this remarkable paper, Sonveaux et al. (11) demonstrate that treatment of tumor-bearing mice with an inhibitor of MCT1 provides a means to kill cancer cells. When MCT1 is inhibited, the metabolic symbiosis is disrupted: aerobic cells can no longer take up lactate, as demonstrated by the analysis of cultured human cancer cells. Instead, these cells increase their uptake of glucose and thereby deprive hypoxic cells of adequate glucose. Thus, MCT1 inhibition in aerobic cells leads to the death of hypoxic cells. Finally, the authors also show that the growth delay of mouse tumor xenografts that is induced by radiotherapy is increased when treatment is combined with MCT1 inhibition (11).

Recent studies reviewed here and elsewhere $(16,17)$ have advanced our understanding of the mechanisms whereby the metabolism of glucose, fatty acids, and amino acids is reprogrammed in human cancers. This metabolic reprogramming is a virtually universal characteristic of advanced metastatic disease. This is best illustrated 
by the observation that the most sensitive clinical test for detecting occult metastases is the uptake of $\left[{ }^{18} \mathrm{~F}\right]$ fluorodeoxyglucose as determined by PET (18). Can a characteristic that is so useful for diagnosis also be exploited for therapy? Research that elucidated the mechanisms by which cancer cells induce blood vessel growth led to the development of angiogenesis inhibitors as anticancer agents over the last decade (19). Inhibitors of HIF-1, LDHA, PDK1, CA9, NHE1, and now MCT1 have shown anticancer effects in tumor xenograft models $(11,17,20)$. It is likely that over the next decade, clinical translation of advances in our understanding of cancer metabolism will have an impact on cancer therapy. Furthermore, a treatment strategy in which tumors are deprived of both $\mathrm{O}_{2}$ (through angiogenesis inhibition) and their ability to adapt to hypoxia (through metabolic inhibition) may represent a formidable combination therapy.

\section{Acknowledgments}

Work in the author's laboratory is supported by the American Diabetes Association; NIH grants R01-HL55338, P20-GM78494, P01HL65608, and N01-HV28180; and the Johns Hopkins Institute for Cell Engineering.
Address correspondence to: Gregg L. Semenza, 733 North Broadway, Suite 671, Baltimore, Maryland 21205, USA. Fax: (443) 287-5618; E-mail: gsemenza@jhmi.edu.

1. Vaupel, P., and Mayer, A. 2007. Hypoxia and cancer: significance and impact on clinical outcome. Cancer Metastasis Rev. 26:225-239.

2. Helmlinger, G., Yuan, F., Dellian, M., and Jain, R.K. 1997. Interstitial $\mathrm{pH}$ and $\mathrm{pO} 2$ gradients in solid tumors in vivo: high-resolution measurements reveal a lack of correlation. Nat. Med. 3:177-182.

3. Semenza, G.L., et al. 1996. Hypoxia response elements in the aldolase A, enolase 1 , and lactate dehydrogenase A gene promoters contain essential binding sites for hypoxia-inducible factor 1. J. Biol. Chem. 271:32529-32537.

4. Kim, J.W., Tchernyshyov, I., Semenza, G.L., and Dang, C.V. 2006. HIF-1-mediated expression of pyruvate dehydrogenase kinase: a metabolic switch required for cellular adaptation to hypoxia. Cell Metab. 3:177-185.

5. Papandreou, I., Cairns, R.A., Fontana, L., Lim, A.L., and Denko, N.C. 2006. HIF-1 mediates adaptation to hypoxia by actively downregulating mitochondrial oxygen consumption. Cell Metab. 3:187-197.

6. Zhang, H., et al. 2008. Mitochondrial autophagy is a HIF-1-dependent adaptive metabolic response to hypoxia. J. Biol. Chem. 283:10892-10903.

7. Ebert, B.L., Firth, J.D., and Ratcliffe, P.J. 1995. Hypoxia and mitochondrial inhibitors regulate expression of glucose transporter-1 via distinct cisacting sequences. J. Biol. Chem. 270:29083-29089.

8. Wykoff, C.C., et al. 2000. Hypoxia-inducible expression of tumor-associated carbonic anhydrases. Cancer Res. 60:7075-7083.

9. Ullah, M.S., Davies, A.J., and Halestrap, A.P. 2006 The plasma membrane lactate transporter MCT4, but not MCT1, is up-regulated by hypoxia through a HIF-1alpha-dependent mechanism. J. Biol. Chem. 281:9030-9037.

10. Shimoda, L.A., Fallon, M., Pisarcik, S., Wang, J., and Semenza, G.L. 2006. HIF-1 regulates hypoxic induction of NHE1 expression and alkalinization of intracellular $\mathrm{pH}$ in pulmonary arterial myocytes. Am. J. Physiol. Lung Cell Mol. Physiol. 291:L941-L949.

11. Sonveaux, P., et al. 2008. Targeting lactate-fueled respiration selectively kills hypoxic tumor cells in mice. J. Clin. Invest. 118:3930-3942.

12. Seagroves, T.N., et al. 2001. Transcription factor HIF-1 is a necessary mediator of the Pasteur effect in mammalian cells. Mol. Cell. Biol. 21:3436-3444.

13. Yun, Z., Maecker, H.L., Johnson, R.S., and Giaccia, A.J. 2002. Inhibition of PPAR gamma 2 gene expression by the HIF-1-regulated gene DEC1/Stra13: a mechanism for regulation of adipogenesis by hypoxia. Dev. Cell. 2:331-341.

14. Liao, D., and Johnson, R.S. 2007. Hypoxia: a key regulator of angiogenesis in cancer. Cancer Metastasis Rev. 26:281-290.

15. Cárdenas-Navia, L.I., et al. 2008. The pervasive presence of fluctuating oxygenation in tumors. Cancer Res. 68:5812-5819.

16. Deberardinis, R.J., Sayed, N., Ditsworth, D., and Thompson, C.B. 2008. Brick by brick: metabolism and tumor cell growth. Curr. Opin. Genet. Dev. 18:54-61.

17. Kroemer, G., and Pouyssegur, J. 2008. Tumor cell metabolism: cancer's Achilles' heel. Cancer Cell. 13:472-482.

18. Gatenby, R.A., and Gillies, R.J. 2004. Why do cancers have high aerobic glycolysis? Nat. Rev. Cancer. 4:891-899.

19. Kerbel, R.S. 2008. Tumor angiogenesis. N. Engl. J. Med. 358:2039-2049.

20. Melillo, G. 2007. Targeting hypoxia cell signaling for cancer therapy. Cancer Metastasis Rev. 26:341-352.

\title{
Autophagy-induced tumor dormancy in ovarian cancer
}

\author{
Ravi K. Amaravadi \\ Department of Medicine and Abramson Cancer Center, University of Pennsylvania, Philadelphia, Pennsylvania, USA.
}

\begin{abstract}
Autophagy - a process of "self-eating" that involves enzymatic digestion and recycling of cellular constituents in response to stress - contributes to both cancer cell death and survival. In this issue of the JCI, Lu et al. report that controlled induction of tumor suppressor gene aplasia Ras homolog member I $(A R H I)$ results in autophagic cell death of human ovarian cancer cells in vitro (see the related article, doi:10.1172/JCI35512). However, within xenograft tumors in mice, multiple factors within the tumor microenvironment switched ARHI-induced autophagy to a mechanism of tumor cell survival, leading to tumor dormancy. Since ARHI expression is suppressed in the majority of breast and ovarian cancers but is high in premalignant lesions, ARHI-induced autophagy could be manipulated for therapeutic benefit.
\end{abstract}

Nonstandard abbreviations used: ARHI, aplasia Ras homolog member I; BECN1, beclin 1.

Conflict of interest: The author has declared that no conflict of interest exists.

Citation for this article: J. Clin. Invest. doi:10.1172/ JCI37667.
Cells that encounter a variety of stresses undergo an evolutionarily conserved process of self-digestion termed autophagy. The importance of this intracellular damage response for pathophysiology has been established across multiple fields, includ- ing infectious disease, neurodegeneration, heart failure, and cancer (1). In cancer in particular, the debate continues to rage as to whether or not autophagy is primarily a mechanism of cell death or cell survival. This is important to understand in order to preferentially promote the clinical development of therapeutic interventions that can either inhibit or enhance autophagy in tumor cells. A number of clinically available cancer therapeutics, including DNAdamaging chemotherapy, radiation therapy, and molecularly targeted therapies have been found to induce autophagy in cell culture and animal models (2). Recent investigations have found that inhibition of therapy-induced autophagy with chloroquine derivatives can enhance cell death in estab- 